\title{
Eye Detection and Tracking and Eye Gaze Estimation
}

\author{
Bhargavi Nadella1)
}

\begin{abstract}
Dynamic Eye-look identification and following have been a dynamic examination held in the previous years as it changes it up of uses. It is viewed as a significant untraditional technique for human PC association. Head development identification has likewise gotten specialists' consideration and enthusiasm as it has been observed to be a basic and successful cooperation technique. Both innovations are viewed as the most straightforward elective interface techniques. They serve an extensive variety of seriously crippled individuals who are left with insignificant engine capacities. For both eye following and head development discovery, a few diverse methodologies have been proposed and used to execute diverse calculations for these advancements. In spite of the measure of examination done on both advancements, specialists are as yet attempting to and powerful routines to utilize viably in different applications. This paper shows a condition of-workmanship study for eye following and head development identification techniques proposed in the writing. Illustrations of distinctive ends of utilizations for both innovations, for example, human computer connection, driving help frameworks, and assistive innovations are likewise examined. Despite dynamic exploration and noteworthy advancement in the most recent 30 years, eye recognition and following stays testing because of the uniqueness of eyes, impediment, and variability in scale, area, and light conditions. Information on eye area and points of interest of eye developments have various applications and are vital in face discovery, biometric recognizable proof, and specific human-PC association errands. This paper audits current advance and best in class in video-based eye recognition and following keeping in mind the end goal to recognize promising strategies and also issues to be further tended to.
\end{abstract}

Keywords : eye, eye following, eye identification, head discovery, eye discovery, look estimation.

\section{Introduction}

Eyes and their developments are essential in communicating a man's longings, needs and enthusiastic states. The significant of eye developments as to the impression of and regard for the visual world is positively recognized subsequent to it is the methods by which the data expected to recognize the attributes of the visual world is accumulated for handling in the human mind[1]. Henceforth, strong eye identification and following are considered to assume a vital part in the advancement of human-PC communication, making mindful client interfaces also, and breaking down human emotional states. Head development is additionally observed to be a characteristic, straightforward and compelling method for indicating items, association

Received(March 16, 2015), Review Result(1st: March 31, 2015, 2nd: April 30, 2015), Accepted(May 10, 2015)

${ }^{1}$ (Corresponding Author) Department of Computer Science and Engineering, KL University email: nadella.bhargavi@gmail.com 
and correspondence[2]. Along these lines, head development identification has gotten noteworthy consideration in late research. One of the different purposes for head development location and following is to permit the client to associate with a $\mathrm{PC}[3]$. It additionally gives the capacity to control numerous gadgets by mapping the position of the head into control signals. Eye following and head development locations are broadly explored as option interface systems[4]. They are considered to be simpler to use than different routines, for example, voice acknowledgment or EEG/ECG signals[5]. Eye developments are the slightest influenced by incapacities in light of the fact that, for instance, spinal string wounds don't influence the capacity to control them, as they are specifically controlled by the mind. Consolidating eye following and head development location can give a bigger number to conceivable control orders to be utilized with assistive innovations, for example, a wheelchair[6-8]. There are numerous methodologies presented in writing centering on eye following. They can be utilized as a base to create an eye following framework which accomplishes the most elevated exactness, best execution and most minimal expense. Head development location has been accepting developing enthusiasm too. There are numerous proposed approaches. Some methodologies may be executed utilizing low computational equipment, for example, a microcontroller because of the straightforwardness of the utilized calculation. This paper shows an overview of distinctive eye following and head development location methods reported in the writing alongside cases of different applications utilizing these advancements. As a standout amongst the most striking elements of the human face, eyes and their developments assume an essential part in communicating a man's goals, needs, subjective procedures, passionate states, and interpersonal relations. The significance of eye developments to the singular's observation of and regard for the visual world is verifiably recognized as it is the strategy through which we assemble the data important to arrange our way through and recognize the properties of the visual world. Vigorous nonintrusive eye recognition and following is, thusly, urgent for the advancement of human-PC cooperation, mindful client interfaces, and comprehension human full of feeling states. The one of a kind geometric, photometric, and movement attributes of the eyes likewise give imperative visual prompts to face location, face acknowledgment, and comprehension facial expressions. For instance, one of the essential stages in the Viola and Jones face finder is a Hair highlight relating to the eye area. This shows the significance of the eyes for face discovery. Also, the separation between the eyes is regularly used for face standardization, for the confinement of other facial milestones, and also in sifting through basic clamor. Look estimation what's more, following are critical for some applications including human consideration investigation, human 
psychological state examination, look based intuitive client interfaces, look unforeseen graphical showcases, and human components. A look tracker is a gadget for dissecting eye developments. As the eye checks the environment or focuses on specific articles in the scene, a look tracker at the same time restricts the eye position in the picture and tracks its development after some time to decide the bearing of look. Research in eye recognition and following spotlights on two regions: eye limitation in the picture and look estimation. There are three parts of eye recognition. One is to distinguish the presence of eyes, another is to precisely decipher eye positions in the pictures, lastly, for video pictures, and the distinguished eyes are followed from casing to outline. The eye position is ordinarily measured utilizing the student or iris focus. The distinguished eyes in the pictures are utilized to gauge and track where a man is looking in 3D, or then again, deciding the 3D observable pathway. This procedure is called look estimation. In the ensuing exchange, we will utilize the expressions "eye identification" and "look following" to separate them, where eye identification speaks to eye limitation in the picture while look following means evaluating look ways. This paper concentrates on eye identification and look following in videobased eye trackers (a.k.a. video-oculography). Video-oculography frameworks acquire data from one or more cameras (Image information). The eye area in the picture is identified and is either utilized straightforwardly as a part of the application or therefore followed over edges. Based on the data acquired from the eye locale and conceivably head represent, the course of look can be assessed. This data is then utilized by look based applications, e.g., moving the cursor on the screen. The main focus of this paper is eye detection and gaze tracking in video based eye trackers which is called a video-oculography. This is mainly done by first detecting an eye and then the eye image is recorded by several placed cameras, which is source of information or data on eye and is called Image data. Then the recorded image is analyzed and the location of eye is detected which is then either used directly in the application or is compared with different frames for tracking.

Gaze estimation information or gaze direction is used by gaze based applications such as moving the cursor on the screen.

Eye tracking and detection methods easier in their usage as compared to other methods such as voice recognition or EEG/ECG signals. In addition to that eye tracking systems also have higher accuracy and performance comparatively. Eye tracking system has also proved as an alternative interface for controlling and communicating, especially for severely disabled people with minimum muscular motions. Eye tracking system also achieves the best performance, highest accuracy and lowest cost. The uniqueness of every individual's eye, its geometric shape 
and motion is use to estimate gaze of an eye and also eye tracking.

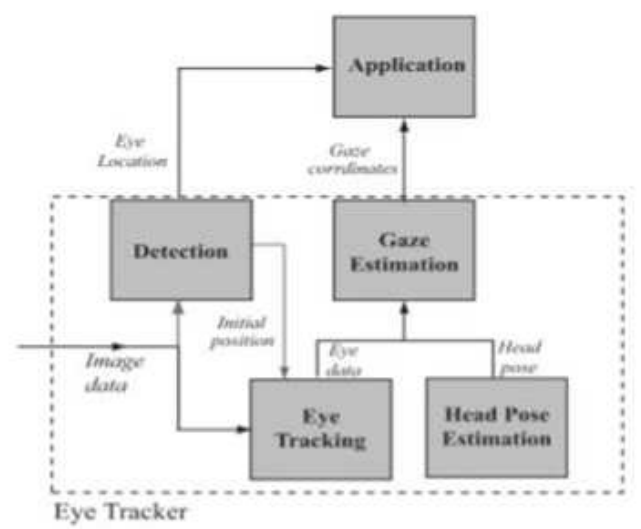

[Fig. 1] Components of video based eye detection and gaze tracking.

\section{Statement of purpose and literature survey}

\subsection{Statement of purpose}

In this paper, the basic questions which are answered based on eye tracking system and eye gaze estimation goes as follows:

Why is the system still facing challenges till date after number of researches?

Why has it become a requirement to study it as a part of human computer interaction?

Whom is the use of eye tracking system helpful to?

In what ways is it helpful in usability testing?

What are the methods involved in the system?

How is it helpful in estimating eye gaze estimation?

How does it act as a combination of software, hardware and biomedical science?

How does it prove to be an interesting research topic?

What are its disadvantages which are still to be succeeded?

What are the aspects of eye detection technique?

To what extent is the system efficient in every aspect?

What are the components involved in establishing the system?

What are the applications of eye tracking system? 
What are the corrections that can be made in the already implemented system?

The paper illustrates, firstly about the system goals in future and achievements till date. It tells us why the system is a requirement especially for human computer interaction and also tells us to what kind of people is the system helpful to. It tells us the system's usefulness in usability testing. The paper explains the estimation of eye gaze and also mentions the methods involved in the implementation of the system. Detailed explanation of the components involved in the system is explained. Advantages, disadvantages, system aspects, its efficiency, its applications and further implementations to be made are discussed in the paper.

\subsection{Literature Survey}

Eye detection and tracking and eye gaze estimation: In the most recent decade, the advancement of eye following (ET) frameworks spoke to a test for analysts and distinctive organizations in the territory of IT, restorative gear or interactive media business gadgets. An eye following framework depends on a gadget to track the development of the eyes to know precisely where the individual is searching and for to what extent. It additionally includes programming calculations for student recognition; picture preparing, information sifting and recording eye development by method for obsession point, obsession length of time and saccade also. A huge assortment of equipment and programming methodologies were executed by exploration gatherings or organizations as indicated by mechanical advancement.

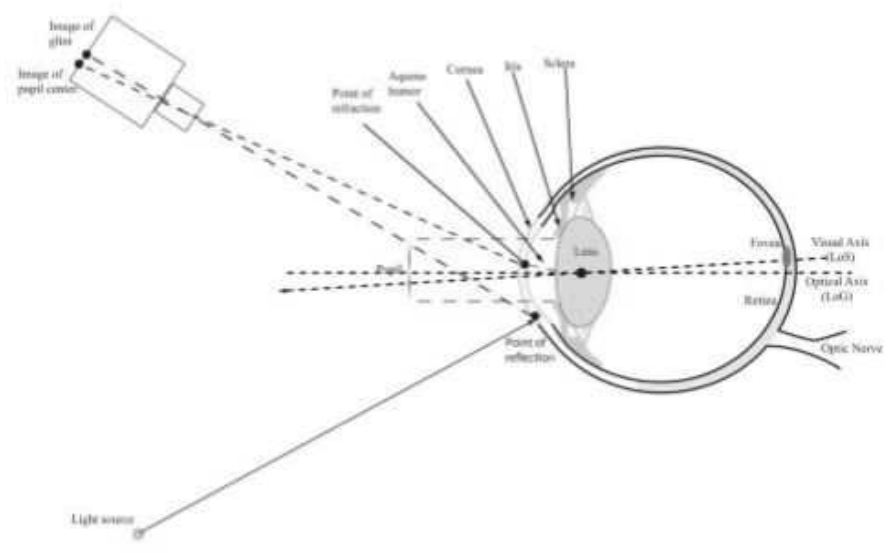

[Fig. 2] General model of the structures of the human eye, light, light sources, and projections. 
The suitable gadgets for eye development gaining and programming calculations are picked in concordance with the application prerequisites. A few sellers (e.g. SensoMotoric Instruments, Tobii or MyGaze) have put resources into eye following innovation, yet their business remote camera-based eye-tracker frameworks for which the light source and camera are forever attached to a monitor. Since these business frameworks including programming and backing are costly some versatile and minimal effort gadgets for eye following were created by some examination bunches. The eye following applications covers human PC association, mind PC communication, assistive innovation, e-learning, brain research examination, pilot preparing help, virtual and increased reality thus on. As innovation advances it is imperative for individuals to have the capacity to stay aware of the advancement or the innovation gets to be outdated. Once the PC turned into a family thing, organizations started inquiring about how to make it easier to understand. Makers of individual gadgets like PCs, telephones, and tablets all contend to make the most advantageous item so they can remain focused of the business sector. This implies they need to stay one stage in front of new innovations. When the touch control framework utilized for the iPhone got to be well known, it rapidly spread to other devices. Voice control is likewise getting to be mainstream in telephones and has as of now been utilized on PCs for debilitated individuals to impart. Organizations are searching for the following best, and least demanding technique for control for individual gadgets. Eye control resembles the most encouraging new innovation that can in the end be utilized economically for some individual purposes. Eye following is a valuable gadget for some regions of study. "We may assume that on the off chance that we can track somebody's eye developments, we can take after along the way of consideration sent by the eyewitness" which will give knowledge into what the client was attracted to and how the client saw and translated whatever he or she saw. This sort of information can be valuable for web originators or publicists on the grounds that they can put content or illustrations in specific spots of the website page where the PC screen would be well on the way to be seen. Does eye-following help with exploration, as well as makes it feasible for debilitated individuals to impart on the off chance that they can't talk or utilize a customary console. Eye following writing interfaces use on-screen consoles that track the onlooker's look to decide the letter they are centering on.

\section{Description}

The one of a kind shapes, shading, structure and development of the eyes helps in giving 
information on eye to performing eye identification and following applications, for example, face location, face acknowledgment and expecting and also understanding outward appearances. The eye locale is additionally one of the elements for face location. One of the striking elements is that the separation between the eyes is utilized for localization of other facial points of interest, face standardization furthermore to filter out basic commotion. This framework is additionally essential for applications like human consideration examination, look based connection client interfaces, human subjective state investigation, look unforeseen graphical presentations another human elements. Data and insights about eye developments, examples, formats, area, and so forth are utilized to distinguish and track eye furthermore gauge eye stare which is consequently utilized as a part of various applications, for example, face recognition, biometric ID and specific human PC cooperation assignments. With expanding advancement in the examination of eye stare estimation and eye following and recognition, new strategies and in addition issues to be talked about are discovered. These frameworks are additionally useful for severely impaired individuals. Survey on eye models, systems to track and recognize eye, strategy to gauge eye stare and their applications are likewise examined on the paper. In this manner all it closes is that, the exploration on eye following and discovery continues forever until it scopes to its most proficient applications. The gadget, look tracker is utilized for breaking down development as a part of eye. As a matter of first importance to gauge eye stare the eye development is recorded and afterward through the investigation of the picture, estimation of eye stare is done, so for this as the eye moves or outputs the earth or whether it is a gaze, settled on a specific article in the scene, look tracker gradually finds the eye position in the picture recorded, to break down and track it development time to time accordingly deciding the look direction.

The primary spotlight on eye following and identification examination are on two zones: Locating eye in a picture Gaze estimation Eye location is partitioned into three perspectives: 1. First to identify if a human arrives to track or recognize an eye. 2. Secondly, to record eye developments in a picture and after that to translate the exact eye positions in the pictures by investigating it. 3. The third viewpoint is that after the investigation of the eye picture, diverse edges are contrasted and the recognized eye.

Understudy or iris focus is for the most part used to quantify eye position. Fundamentally the recorded picture of a man's recognized eye is utilized to gauge and track a man's 3D perspective or $3 \mathrm{D}$ viewable pathway and is called look estimation. The distinction between "eye recognition" and "look following" is that eye discovery is utilized to represent the area of eye in the recorded picture while look following is utilized to gauge the $3 \mathrm{D}$ viewable pathway of a 
man. The principle center of this paper is eye discovery and looks following in video based eye trackers which are known as a video-oculography. This is predominantly done by first recognizing an eye and afterward the eye picture is recorded by a few put cameras, which is wellspring of data or information on eye and is called Image information. At that point the recorded picture is investigated and the area of eye is recognized which is then either utilized straightforwardly as a part of the application or is contrasted and distinctive edges for following.

The diverse parts of eye following in its different uses involves:

1. Eye following of sites and programming: The regular perception strategies for eye-following information are Heat maps and output ways. Quick reaction of clients and their consideration appropriation in an association is appeared by eye following, as:

- What is the most consideration looking for point of view in a site?

- Where ought to the imperative matter be set?

Furthermore, when comes to taking care of the issue, after the entire parcel of its examination, eye following gives complete data around a client cooperation with an interface.

- Is the structural planning of the site or programming reasonable by the clients?

- Do the images spoke to, portrays its utilization flawlessly?

- What is important are just to look over at and what is to be tapped on?

- What does the choice making procedure similarize with?

The most straightforward structure to test the utilization of eye following is live review. It is likewise a quick strategy for clear comprehension between a client and an interface. Particularly with regards to a site, watching the very same thing what the client sees will enhance the site and unravel the problems.

2. Eye following of recreations: Eye following is likewise helpful in the investigation of client cooperation with amusements and TV. It is valuable in the change of way discovering, deceivability of visual narrating or battlefield, menu route and instructional exercise texts.

3. Eye following of cell phones: It gives information or data about the cooperation of clients 
with a portable GUI or other equipment components which help expert's to prescribe select compelling outlines.

- How does a client uses a portable application?

- Where does a client discover catches of alternatives in a physical gadget?

- How does the consideration moving of a client happens between distinctive parts of a gadget?

To think about subjective eye following and for bigger gadgets, portable eye tracker can be utilized as a result of its free development. An emulator can be utilized for testing portable interfaces on screen. These emulator's works like virtual mobiles on PC screen.

4. Eye following of in-field gadgets: These eye following frameworks are smaller and snap-on and is utilized for certifiable interfaces that doesn't offer space to fit a fullsized remote eye tracker. For example it can be utilized to concentrate on in-field gadgets, for example, ticket machine or registration machine.

5. Eye following in true situations: In true situations, for example, airplane terminals and shopping centers, versatile eye following arrangements give information about the signage or computerized showcases use.

- To know the effectiveness of signage in directing explorers to baggage carousel territory, telephones, sustenance courts, takeoff door and different necessities?

- Icons supplant words in what cases?

It permits client to connect unreservedly with physical gadgets, for instance, nourishment blender, candy machine or control board in a car. Presently these were a portion of the convenience testing of eye following which with the assistance of eye perception and recording aides being developed and improvement of numerous human-PC cooperation applications and gadgets. With this the proficiency of uses and gadgets can be enhanced, as it were, and this issue can be settled utilizing the eye following framework. Another of the significant information or confirmations on which the issue of eye following is based is security purposes, exceptionally for handicapped individuals furthermore for other business and expert security purposes, whether they are prominent secret workplaces, banks or different spots where 
security is an absolute necessity, eye following framework plays a vital role. As indicated by the analysts on the off chance that we watch deliberately, we can reason that the eye following applications were produced or designed to beat certain issues which were settled utilizing eye following framework and these applications end up being authentic proofs and inductions for taking care of the issues. So on the off chance that we consider the eye following exploration field, it has been extremely dynamic on account of its number of uses.

A percentage of the utilizations of eye following frameworks which takes care of a percentage of the issues are:

EYE CONTROL FOR ACCESSIBILITY AND ASSISTIVE: Individuals who have lost the control over every one of their muscles and are no more ready to perform willful developments as a aftereffect of infections or mishaps can Benet generally from eye following frameworks to collaborate and speak with the world in everyday life. Eye tracker frameworks give numerous alternatives to these people, for example, an eye-writing interface that could have content to-discourse yield. They additionally empower eye-control, counting coordinating electric wheelchairs or exchanging on the Television or different gadgets. Human PC collaboration with graphical client interface activities or occasions may be classified into two primary classifications: Pointing: Moving the pointer over an item on the screen for example, content or symbol on the screen. Selection: Activity deciphered as a determination of the article indicated. Presented the "Eye Mouse" which with extreme inabilities can utilize. The mouse cursor position is resolved in light of the obtained data about the eye position towards the screen which gives the capacity to work a PC by individuals with serious handicaps. Devoted their eye following framework to the help of individuals with incapacities. The framework was utilized with three on-line applications. The rest controls a versatile robot in a labyrinth. The second application was "Eye Essayist" which is a content written work program. A PC diversion was the third application. Utilizing data acquired from following the eye stare to control a presentation based on video. Eye stare is assessed and the presentation is controlled in like manner. "Asistsys" which is a correspondence framework for patients experiencing neuro-locomotor incapacities. This framework helps patients in communicating their needs or longings. E-LEARNING: E-learning frameworks are PC based showing frameworks and are presently extremely basic. In any case, regardless of the way that clients are generally usual to machine cooperation's, the learning experience can be entirely diverse. Specifically, the "emotional" part is significant in the cooperation between educator what's more, learner and it is lost in PC based learning forms. "e5Learning" which is an e-learning environment that 
adventures eye information to track client exercises, practices and enthusiastic or "affective" states. Two fundamental client states were considered: "high workload or nonunderstanding" furthermore, "tiredness." The creator/instructor of the course has the capacity choose the length of time the client ought to take a gander at certain parts of the course content, whether this substance was literary or non-printed. It tried to manufacture an elearning stage which figures out if an understudy is having difficulty seeing some substance or is drained or focused on taking into account the translated eye conduct. Exploration is done on applying eye following routines in the vehicle industry with the point of creating checking and helping frameworks utilized as a part of autos. For instance, an eye tracker could be utilized as a part of autos to caution drivers when they begin getting tired or nod off while driving. Driver weariness can be recognized by breaking down squint limit, eye state (open/shut) and for to what extent the driver's look stays in the same bearing. Numerous eye following routines were utilized as a part of this region of use.

IRIS RECOGNITION: Iris acknowledgment is as a rule broadly utilized for biometric verification. Iris limitation is an essential and basic step whereupon the execution of an iris acknowledgment framework depends.

FIELD OF VIEW ESTIMATION: Another intriguing use of eye following frameworks is that these frameworks can serve as a viable apparatus in optometry to help with recognizing the visual end of any person, particularly recognizing blind sides of vision utilized eye following to evaluate the end of perspective to be utilized for increased video/picture/illustrations show.

\subsection{Eys Detection and Eye Tracking Techniques}

EYE MODELS FOR EYE DETECTION: In eye distinguishing proof, it is key to perceive a model of the eye which is sufficiently expressive to make note of considerable variability in the appearance and movement, while too satisfactorily constrained to be computationally gainful. The presence of eye regions offers shared qualities transversely over race, edification, and overview edge, in the meantime, despite for the same subject, a for the most part little assortment in review focuses can achieve vital changes in appearance. Despite element examination, eye area and taking after remains an incredibly troublesome undertaking in light of a couple of novel issues, tallying obstruction of the eye by the eyelids, level of openness of the eye, variability in either size, reflectivity or head stance, et cetera. Uses of PC vision, for instance, people taking after, face area, and diverse restorative applications, experience obstructions and shape assortments, yet, on occasion of the same solicitation of size and repeat 
as seen with the eyes. The eye picture may be depicted by the power transport of the pupil(s), iris, and cornea, and furthermore by their shapes. Ethnicity, audit point, head stance, shading, surface, light conditions, the position of the iris within the eye connection, and the state of the eye (i.e., open/close) are issues that igorously affect the vicinity of the eye. The proposed application and available picture data lead to various prior eye models. The prior model representation is every now and again associated at unmistakable positions, presentations, and scales to reject false candidates. Being either inflexible or deformable, the exploratory order of eye acknowledgment systems includes shape-based, appearance-based, and creamer schedules. Shape-based frameworks can be subdivided into settled shape likewise, deformable shape. The frameworks are fabricated from either the area point parts of the eye and face region or from their shapes. The opportune components may be edges, eye corners, or centers picked in light of specific channel responses. The limbus and understudy are by and large used highlights. While the shape-based schedules use a before model of eye shape and incorporating structures , the appearance-build systems depend regarding models construct direct in light of the vicinity of the eye locale. The appearance-based philosophy (the sweeping approach) sensibly relates to arrangement developing in order to facilitate a photo patch model and performing eye acknowledgment through model organizing using a closeness measure. The appearance-based frameworks can be further isolated into power and subspace-based frameworks.
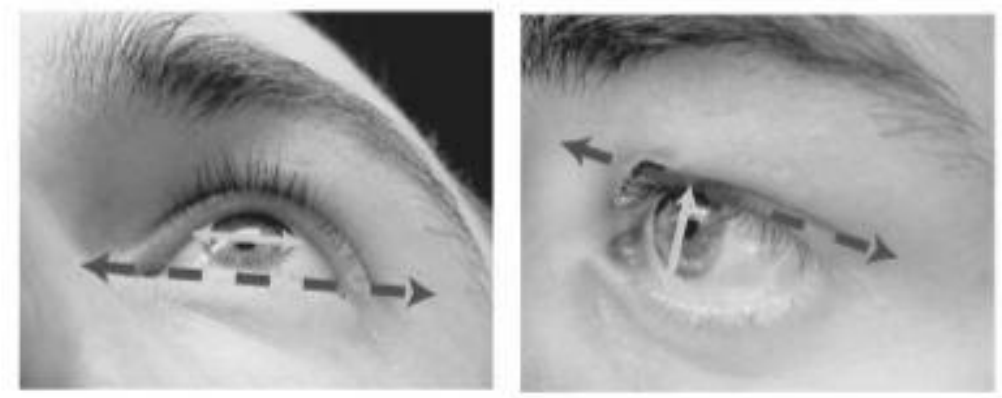

[Fig. 3] The shape of the eye may change drastically when viewed from different angles.

For example, the eyelids may appear straight from one view but highly curved from another. The iris contour also changes with viewing angle. The dashed lines indicate when the eyelids appear straight, while the solid yellow lines represent the major axis of the iris ellipse.

Shape-Based Approaches: The open eye is all that much depicted by its shape, which 
consolidates the iris and understudy frames and the outside condition of the eye (eyelids). Course of action of shape-construct philosophies depends in light of whether the prior model is essential bended or of an additionally astounding nature. Shape models as a rule constitute two sections: a geometric eye model and a likeness measure. The parameters of the geometric model portray the suitable design deformations and contain parameters for firm (equivalence) changes and parameters for nonrigid configuration contortions. Deformable shape models routinely rely on upon a tasteless deformable organization by which the eye is arranged by distorting the shape model through an essentialness minimization. A basic property of these frameworks is their general ability to handle shape, scale, besides, changes.

Simple Elliptical Shape Models: Various eye taking after applications simply require the discernment and taking after of either the iris or the understudy. Dependent upon the audit point, both the iris and understudy appear to be round and along these lines can be shown by five shape parameters. Straightforward circle models include voting-based methods and model fitting schedules. Voting procedures select segments that support a given hypothesis through a voting or conglomeration process, while model fitting techniques fit picked components to the model (e.g., oval). Fundamental shape models are regularly capable and they can display components, for instance, iris and understudy well under various overview focuses.

Complex Shape Models: Complex shape-based frameworks license, by definition, for clearer exhibiting of the eye shape. The model is fitted to the photo through a redesign standard, which joins imperativeness limits for valleys, edges, picture peaks, and internal qualities. Exploratory investigation finds that the beginning position of the design is essential. Deformable format based strategies give off an impression of being genuine and are generally correct and non particular, be that as it may they encounter the evil impacts of a couple of imperatives. They are:

1. computationally asking,

2. may require high separation pictures, and

3. regularly ought to be instated close to the eye for productive limitation. For limitless head improvements, they consequently require distinctive schedules to give an incredible presentation.

4. Deformable shape models may go up against besides issues while using IR light as the cutoff of the sclera and the face may appear to be feeble. 
5. They will be not able to handle face stance changes moreover, eye obstacles well. While some deformable models, for instance, snake models, consider much shape variability, other deformable models do whatever it takes not to consider the enormous variability of eye shapes. Further research is relied upon to make models that can adjust with inconceivable shape assortments and even handle misshapenness for instance, key conclusion or clashing part region (i.e., segments showing up and vanishing with changes in scale).

- Feature-Based Shape Methods : Highlight based frameworks examine the characteristics of the human eye to recognize a plan of unmistakable components around the eyes. The limbus, understudy (faint/splendid understudy pictures), and cornea reflections are typical segments used for eye constraint. Appeared differently in relation to the complete approachs, highlight based schedules hope to perceive informative close-by segments of the eye and face that are less delicate to assortments in illumination and point of view.

- Appearance-Based Methods : While the condition of the eye is a basic descriptor, so is its appearance. The appearance-based frameworks are in like manner known as picture arrangement or widely inclusive procedures. The appearance-based frameworks perceive and track eyes particularly, considering the photometric appearance as portrayed by the shading spread or channel responses of the eye and its surroundings. These methods are self-governing of the certifiable object of interest and are, on an essential level, prepared for showing different challenges other than eyes. The term appearance may be gotten on as one or a couple pictures (designs) described point wise with appearance given by the movements of force or their channel responses. The appearance-based approaches are finished either in the spatial or in a changed space. One of the rule points of interest of performing eye revelation (object recognizable proof by and large) in a changed space is to alleviate the effect of edification assortment by protecting sub bands that are less sensitive to lighting up and evacuating gatherings that are fragile to lighting up change. Such routines, nevertheless, arek eventually only tolerant to direct lighting up change. Appearance-based frameworks can be picture template based, where both the spatial and strained quality information of each pixel are spared or widely inclusive in system, where the power apportionment is depicted by ignoring the spatial information. Picture arrangement based procedures have trademark issues with scale and rotational changes.

\section{Discussion}


In this segment, we abridge distinctive methods for eye identification and following. Taking into account their geometric and photometric properties, the strategies can be delegated shape-based, element based, appearance-based, and cross breed. Elective methods may endeavor movement and symmetry. Dynamic IR brightening may be utilized by different methods. Every method has its favorable circumstances and confinements; be that as it may, the ideal execution of any method too infers that its specific ideal conditions as to picture quality are met. These conditions identify with brightening, head stance, ethnicity, and level of eye impediment. For case, the methods in light of dynamic IR lightenment function admirably inside, while methods in light of shape and appearances can work sensibly well both inside and outside. The current routines are to an expansive degree just appropriate to close frontal perspective points, completely open eyes, and under generally obliged light conditions. Likewise, the eye appearance may change essentially with changes in scale. Elements characterized on one scale don't exist or have changed drastically in another scale. It is thusly testing to apply a solitary scale eye model to various scales. It would in this manner be educational to decide conveyances of elements and highlight reactions for the class of eyes, as in regular picture measurable methodologies, in order to be better ready to control for changes in eye appearance over scale. It remains a test to identify and track the eyes due to wide, complex varieties in the eye picture properties due to ethnicity, light conditions, scale, head posture, and eye state (open/shutting eyes). As of late, fix based systems for item identification, acknowledgment, and arrangement have gotten huge consideration as they show promising results. As an element based strategy, they have a tendency to be more discriminative, more hearty to face stance, and light variation than the all encompassing eye identification approaches. The expectation is that peruses can decide suitable strategies for their specific applications. Before finishing up this segment, we likewise need to talk about a few related issues: 1) with a specific end goal to create successful eye identification strategies, the preparation and testing of eye information are fundamental. Different eye and face databases. The eye picture necessities contrast among the systems examined in this segment. While equipment decision plays an imperative part, we have so far abstained from putting an excessive amount of accentuation on these issues, yet rather decided to depict hardware independent eye recognition procedures. A few applications utilize genuinely top notch cameras with variable lenses and some of the time with dish and tilt heads so as to perform precise and strong eye recognition under states of substantial head developments. These applications acquire high cost. On the other hand, a few applications intend to utilize low-quality purchaser cameras to minimize expense to the customer. Lowcost arrangements with a standard lens may 
require the camera to be near the eye and the head to be moderately stationary. The appearancebased systems of both force and subspace areas have been generally connected to face location, creature identification (e.g., stallions), furthermore, vehicles. The neighborhood highlight based systems have been connected to the location and following of other facial components including mouth corners and nose corners. The essential undertaking of look trackers is to decide look. Look should in this setting be comprehended as either the look heading or the purpose of respect (PoR). Gaze demonstrating thusly concentrates on the relations between the picture information and the purpose of respect/look heading. Fundamental classifications of eye developments incorporate saccades what's more, obsessions. An obsession happens when the look rests for some base measure of time on a little predefined territory, generally inside of 2-5 degrees of focal vision, as a rule for at minimum 80-100 ms. Saccades are quick, bounce like pivots of the eye between two focused territories, bringing objects of hobby into the focal couple of degrees of the visual field. Smooth interest developments are a further arrangement that portray the eye taking after a moving article. Saccadic eye developments have been broadly researched for a wide scope of uses including the location of weakness/ sleepiness, human vision studies, diagnosing neurological disarranges, and rest examines. Obsessions are regularly broke down in vision science, neuroscience, and mental studies to decide a man's center and level of consideration. Properties of saccades and obsessions may give symptomatic information for the recognizable proof of neurological, vision or rest scatters. Squint location might be accomplished through moderately basic measures of the eye area (e.g., format connection or the variety from the force mean). Notwithstanding, quick squinting and head developments make dependable flicker discovery testing. Keeping track of eye qualities amid flickers may be vital, therefore, non eye elements may be more dependable (e.g., utilizing between-the-eyes layouts). Besides, eye recognition in light of squinting is presently restricted to identifying eyes in close frontal appearances. One conceivable answer for distinguishing flicker amid head developments is to track the movement of a couple inflexible element focuses on the face, and subtract their movement from that of the eye movement to minimize the impact of the head development.

\section{Conclusion}

Eye following and head development identification are viewed as successful and solid human-PC collaboration and correspondence elective techniques. Thus, they have been the subject of numerous exploration works. Numerous methodologies for actualizing these 
innovations have been accounted for in the writing. This paper researched existing techniques and exhibited a condition of-workmanship review on eye following and head development identification. Numerous applications can underneath from using viable eye following and/or head development discovery techniques. Nonetheless, the examination is as yet confronting difficulties in exhibiting powerful systems which can be utilized as a part of utilizations to identify and track eye or head developments precisely. Eye following strategies once in a while research the required CPU time. Nonetheless, ongoing application requires exploring and streamlining the execution prerequisites. Also, most studies don't test eye following utilizing a known picture database that contains variation pictures of diverse subjects in distinctive conditions, for example, lighting conditions, commotion, separations, and so forth. This makes the reported precision of a technique less dependable in light of the fact that it might be influenced by distinctive test conditions. We have introduced a survey arranging eye following frameworks from various points; from the diverse routines for recognizing and following eye pictures to computational models of eyes for look estimation and look based applications. In particular, for eye recognition and following, we have talked about different methods utilizing distinctive properties of the eyes including appearance, shape, movement, or some mix. While these strategies have been fruitful in enhancing eye location and following, there stays huge potential for further developments.

\section{Referenses}

[1] A. Al-Rahayfeh and M. Faezipour, Eye Tracking and Head Movement Detection: A State-of-Art Survey, IEEE Journal of IEEE Journal of Translational Engineering in Health and Medicine, (2013), Vol.1.

[2] J. G. Wang, E. Sung, and R. Venkateswarlu, Eye Gaze Estimation from a Single Image of One Eye, Proceedings of the Ninth IEEE International Conference on Computer Vision (ICCV 2003) 2-Volume Set, (2003).

[3] D. W. Hansen and Q. Ji, $\mathrm{n}$ the Eye of the Beholder: A Survey of Models for Eyes and Gaze, IEEE Transactions on Pattern Analysis and Machine Intelligence, (2010), Vol.32, No.3, pp.478-500.

[4] K. P. White Jr., T. E. Hutchinson, and J. M. Carley, Spatially Dynamic Calibration of an Eye-Tracking System, IEEE Transactions on Systems, Man, and Cybernetics, (1993), Vol.23, No.4, pp.1162-1168.

[5] O. Williams, A. Blake, and R. Cipolla, Sparse and Semi-supervised Visual Mapping with the $\mathrm{S}^{3} \mathrm{GP}$, Proc. IEEE CS Conf. Computer Vision and Pattern Recognition, (2006), pp.230-237.

[6] X. Xie, R. Sudhakar, and H. Zhuang, A Cascaded Scheme for Eye Tracking and Head Movement Compensation, IEEE Trans. Systems, Man, and Cybernetics, (1998), Vol.28, No.4, pp.487-490. 
Eye Detection and Tracking and Eye Gaze Estimation

[7] http://www.sandia.gov/nisac/fait.htm, March 23 (2012).

[8] http://www.sandia.gov, Febrary 29 (2012). 